International Journal of Electrical and Computer Engineering (IJECE)

Vol. 11, No. 5, October 2021, pp. 4174 4182

ISSN: 2088-8708, DOI: 10.11591/ijece.v11i5.pp4174-4182

\title{
Elastic hybrid MAC protocol for wireless sensor networks
}

\author{
Jamila Bhar ${ }^{1}$, Imen Bouazzi ${ }^{2}$ \\ ${ }^{1}$ NOCCS Laboratory, ENISo, University of Sousse, Sousse, Tunisia \\ ${ }^{2} \mathrm{E} \mu \mathrm{E}$ Laboratory, FSM, University of Monastir, Tunisia
}

\begin{tabular}{l}
\hline \hline Article Info \\
\hline Article history: \\
Received Aug 11, 2020 \\
Revised Mar 21, 2021 \\
Accepted Apr 6, 2021 \\
\hline
\end{tabular}

Keywords:

Access protocols

Data transfer

Elastic

MAC

Wireless sensor networks

\begin{abstract}
The future is moving towards offering multiples services based on the same technology. Then, billions of sensors will be needed to satisfy the diversity of these services. Such considerable amount of connected devices must insure efficient data transmission for diverse applications. Wireless sensor network (WSN) represents the most preferred technology for the majority of applications. Researches in medium access control (MAC) mechanism have been of significant impact to the application growth because the MAC layer plays a major role in resource allocation in WSNs. We propose to enhance a MAC protocol of WSN to overcome traffic changes constraints. To achieve focused goal, we use elastic hybrid MAC scheme. The main interest of the developed MAC protocol is to design a medium access scheme that respect different quality of services (QoS) parameters needed by various established traffic. Simulation results show good improvement in measured parameters compared to typical protocol.
\end{abstract}

This is an open access article under the CC BY-SA license.

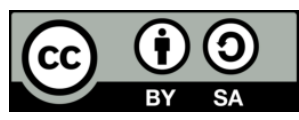

Corresponding Author:

Jamila Bhar

NOCCS Laboratory, ENISo

University of Sousse

Sousse, Tunisia

Email: jamila.bhar@issatso.u-sousse.tn

\section{INTRODUCTION}

The wireless sensor network protocols are sensitive to routing algorithms, location-based protocols, mobility, topology and QoS metrics. To maintain a sensor network running in perfect state, many constraints must be fulfilled in account as applications type, sporadic traffic, time synchronization, sensor lifetime and energy consume. Hence, to achieve availability, scalability and reliability for various considered conditions, there is a crucial need for an efficient MAC protocol. The behaviour of a channel access method is essential selection to take for the development of MAC protocol. Usually, MAC unreliability depends on suitability of the contention mechanism or priority method for traffic conditions. Major heavy traffic environments use asynchronous multiple access and then apply carrier sense multiple access/collision avoidance (CSMAICA) protocol. But, for dense networks, time division multiple access (TDMA) scheme becomes more preferred. Joining the two methods is a hard challenge to surmount that requires great attention. Different methods can be proceeded to maintain a good performance level. QoS ways to improve these schemes are generally based on prediction [1], fuzzy [2], routing [3] and others decisive factors. A comparative analysis of various QoS MAC protocols is presented in [4] to survey their strengths and limitations. Scheduling, polling, adapting, and prioritizing are the most approaches considered to enhance MAC protocols in WSN environment especially for resolving internet of things (IoT) problems. Mac protocol improvement is remarkable for hybrid MAC scheme as it combines the strong points of multiple MAC protocols. Even though, switching between different MAC protocols presents some complexity as synchronization and decision strategies. Finding MAC protocol suitable for heterogeneous sensor networks circumstances, still need more intensive 
research. In general, suggested solutions depend on particular defined properties, which make application areas limited. Hence, a promising research in testing WSN MAC protocol performances for various traffic conditions needs to be studied more extensively. The aim of the present study is to develop solution, which improves MAC protocol to deal with channel sharing problems especially for sporadic traffic. In order to fulfil the performances requirements of applications, this work focuses on a solution that brings significant advantages such as equitable transmission, minimum packets drop and high delivery ratio. The challenge of ensuring these advantages is usually faced by the resource constrained nature of the WSN like limited network lifetime, consuming energy and sharing channel. The essential improvement proposed for this MAC protocol is the dynamic aspect to well adapt the existing bandwidth to active sources. It focuses to use contention method for low traffic and to apply priority schedule method for dense traffic so that the sensor alters between CSMA/CA and TDMA transmission scheme as the traffic circumstances require. This paper is organized is being as. Section 2 explores the improvement of MAC protocol cited in the literature. Details of the proposed contribution of an elastic hybrid MAC protocol are given in section 3. Simulation results are shown in section 4. Lastly, section 5 concludes this paper.

\section{IMPROVEMENT OF WSN MAC PROTOCOLS}

Developed MAC protocols can be classified referring to diverse aspect. Firstly, channel access can use single channel with limited bandwidth or multi-channel that give parallel data flow [5]. In single channel case, CSMA/CA offer scalable communication for low traffic and it can use feedback control with RTS/CTS mechanism [6]. While TDMA present a good reliability for dense traffic and it can guaranty priority access. Hybrid protocol uses a traffic adaptive access based on TDMA/CSMA approaches and guaranty priority channel access for critical applications. A strategy that alters between three schemes explained above is a solution that adapts channel access to traffic conditions changes. Several enhancements can be attained such as throughput, traffic emergency support and coexistence of diverse application types as the approach can support both periodic and on-demand channel access.

To ameliorate the communication procedure, many traffic adaptive MAC protocols have been proposed as TMAC, ZMAC, PMAC and DMAC. TMAC protocol uses adaptive duty cycle. It has the problem of early sleep that allow node to lose synchronization, but it can offer higher energy efficiency than SMAC [7]. In ZMAC, a node senses the channel and only transmits a packet if it is sensed clear. However, the owner of the slot always has higher priority than other nodes in accessing the channel [8]. PMAC is good for delay sensitive application. It uses hybrid strategy to attempt high throughput in dense traffic [9]. DMAC is a contention-based protocol that gives good delay performances and energy saving due to sleep schedules [10]. As mentioned above, the protocols SMAC and TMAC are suitable for high traffic applications, PMAC for delay sensitive applications, ZMAC for large range of applications and DMAC for priority-based applications. Therefore, they give different solutions to accommodate heterogeneous traffic since slots allocation is assigned according to the traffic volume. So, performances of each protocol listed above depend on preamble case, priority level and/or sleep schedule strategy that influence energy consumption. They are as well sensitive to traffic conditions. Slama et al. [11] show a significant improvement in performances of an adaptive hybrid IMAC protocol compared to ZMAC. IMAC access protocol allows sensor nodes that have higher load to get more chances to access the radio resources. This is achieved through an adaptive prioritization mechanism embedded within the transmission mechanism that shortens their listening period (backoff) and reduces the collision rate. Since the WSN topology is a star topology and most of the traffic is uplink, WSN is based on topology management using adaptive clustering techniques. Clustering is an intelligent method to enhance MAC protocol performances [12]. Low energy adaptive clustering hierarchy $(\mathrm{LEACH})$ is a good protocol for this network type [13]. The protocol aims to support large scale WSNs by intelligently grouping nodes into hierarchies of clusters using separate channels rather than using a single high-capacity channel. LEACH employs a hybrid TDMA/CSMA multiple access technique, much like the beacon enabled mode of IEEE 802.15.4. Due to the use of this technique, synchronization requirements and CSMA/CA drawbacks are challenges faced in the protocol [14].

MAC protocol handles burst traffic mainly by using the variable TDMA slots reserving. Generally, coordinator node attaches a suitable number of TDMA slots to each concerned node for packet transmission in the next superframe according to its queue length information and a number of demands of slot allocation. Zhuo et al. [15] proposed hybrid CSMA/TDMA MAC protocol called iQueue-MAC for dynamic and busy traffic in order to mitigate packet buffering and reduce packet delay. The protocol alters to a contention mechanism to provide low delay if the traffic in a system is low and it uses a scheduled mechanism allocating transmission slots if traffic is high. iQueue-MAC is able to effectively use multiple channels, duplicating its throughput when compared to single channel operation. Another two ideas are proposed in [16] to improve the traffic efficiency by IEEE 802.15.4 MAC layer optimization. The first idea is to adjust the backoff 
exponent dynamically based on the queue level of each node. The next idea is to alter the amount of successive clear channel assessment for data packet transmissions. This approach minimizes the resource wastage in the network. Pattamasiriwat and Jaikaeo [17] present a low power listening (LPL) mechanism, in which the period of listening is reduced. If the channel is idle the nodes go into sleep mode, otherwise they stay on the channel to receive the data. The periodic sampling is efficient for high-traffic nodes and performs well under variable traffic conditions. However, it is ineffective for low traffic nodes, especially for periodic traffic.

The use of hybrid MAC protocol is an important solution that can solve the problem of the data traffic that can suddenly going from high to low and vice versa. A decision whether to transmit using contention period, or transmit using contention free period, or both, or not to transmit at all is explained in [18]. Authors propose a scheme to enhance hybrid CSMA/CA-TDMA procedure in congested networks case based on markov decision process (MDP). Simulation results of this access model outperform the CSMA/CA scheme. A game theory based hybrid MAC (GH-MAC) protocol for intra and inter cluster domain is presented in [19]. Authors show that the proposed protocol can enhance the lifetime of WSN and enormously reduce the energy consumption of the nodes by adapting the level of contention in the network either high or low. A hybrid TDMA/CSMA MAC protocol for event-driven sensor networks is proposed in [20]. It is an adaptive duty cycle MAC protocol, called pQueue-MAC, which achieves high energy efficiency and, in the meantime, guarantees immediate data transmission. A TDMA/CSMA hybrid MAC layer protocol (TCHMAC) can enhance the energy efficiency and the transmission throughput of WSNs [21]. The proposed model handles traffic bursting problem by using traffic adaptive TDMA (TA-TDMA) scheme and energy efficient CSMA (E-CSMA) scheme in the protocol. Petrosky et al. [22] present an overview of multiple access techniques and explain how RA-CDMA characteristics can outperform other techniques.

Referring to various protocols explained in literature each proposition is adequate to designed traffic conditions or to enhance particular parameters performances. A classification of proposed MAC protocols may be necessary to compare their characteristics. It is widely marked in literature that the main causes of energy waste are idle listening, overhearing, overhead and especially collision [23], [24]. Researchers usually consider scheduling nodes by sleep and wake periods as a successful way used in wireless sensor networks to save energy. Iala et al. [25] present a solution for reducing the power consumption of WSN thanks to a process that wakes nodes up only if they should transmit or receive data packets. We suggest that to decrease energy dissipate causes and to help nodes to operate properly for long duration of time, medium access strategy must truck network parameters for every superframe to distribute slots equitably. A survey presented in [26] refers the causes of decrease in lifetime of WSN to the significant increase of energy consumption of nodes nearer to the sink. The solution proposed is to balance load of the sensor nodes in the bottle neck zone.

To summarize, an extensive review and various criteria are considered for classification of Mac protocols. Mac protocol performances can greatly improve by using intelligent sleep/wake-up procedure as explained in [27]. The idea of optimizing CSMA algorithm is also a successful way to reduce transmission delay especially handoff time. Gamal et al. [28] use a markov model to reduce the average delay and improve reliability compared to the standard algorithm. Enhancement is less remarkable when node number increase. The problem of medium-access decision within a dense network composed of nodes with low duty-cycles is referred in [29]. Authors give a classification of MAC protocols introduced in literature and giving efficient optimization considering support of conditions change. A taxonomy of MAC protocol is explored in [30] for the comparison of surveyed MAC protocols for WSNs. A detailed overview of recently proposed MAC protocols developed for prioritized heterogeneous traffic in WSN is also explained in [31]. It classifies the recent priority-based MAC protocols in the categories of adaptive contention, duty-cycle adaptation, queue management and hybrid. All research proposed in literature gives numerous enhancements for MAC protocol by different strategies. There is no solution method of comparing the performance of optimized MAC protocols, even for protocols belonging to the same category. The lack of standard evaluation metrics made it difficult to evaluate and select a protocol, even if the requirements of a particular application are known especially with the rapid expanding of wireless sensor network protocols. Analysis method and metrics presented in this paper suggests that elastic hybrid based approach may be helpful when the network conditions are random and application requirements are heterogeneous. Analysis also shows that prioritybased approaches may be more efficient to manage network balance.

\section{ENHANCED MAC PROTOCOL PROPOSAL}

Based on the analysis of application requirements and the literature survey, we conclude that hybrid MAC mechanisms are found to be efficient solutions for WSNs that maintain good throughput, reduce delay transmission efficiently and enhance energy saving significantly. In this way, this contribution to ameliorate MAC protocol of WSN environment aim to attempt challenges of short queue delay, high PDR and less 
frames drop. The proposed elastic hybrid (E-hybrid) MAC mechanism works in two modes, contention and priority schedule modes. We consider that the access to the channel is dynamically allocated based on CSMA and TDMA according to the requirement of the application. The decision is made by a constraints control mechanism. The developed constraints control mechanism evaluates traffic conditions and decide about the desired GTS slots number to be reserved for next superframe. The work assumes that all nodes initially work in typical hybrid mode. The shift to E-hybrid protocol is based on condition related to a collision, priority and traffic density. We consider also that if there is no slot reservation for CFP period, nodes can allow these free slots to complete transmission. The main defy of the present proposal is to balance efficiencies of all evaluated QoS parameters. In consequence, E-hybrid scheme needs to dynamically switch distribution of the three alternative strategies on the superframe according to changes in system conditions. Then, it extends contention period in low traffic condition. It enlarges reserved slots to scheduling algorithm when data traffic becomes high and it perform emergent CFP period at the beginning of the superframe for urgent data. This channel access strategy may compensate any picks of use of resources and optimize the performances of QoS metrics. Figure 1 depicts different procedures integrated in the proposed strategy.

Collect information from nodes (over separate circuit other then used for data transfer)

\section{Constraints control mechanism}

Information classification

INPUT (trafic rate, FIFO state, Persistant congestion and

Emergency case)

OUTPUT (Priority level (Urgent, High, Medium or Low)

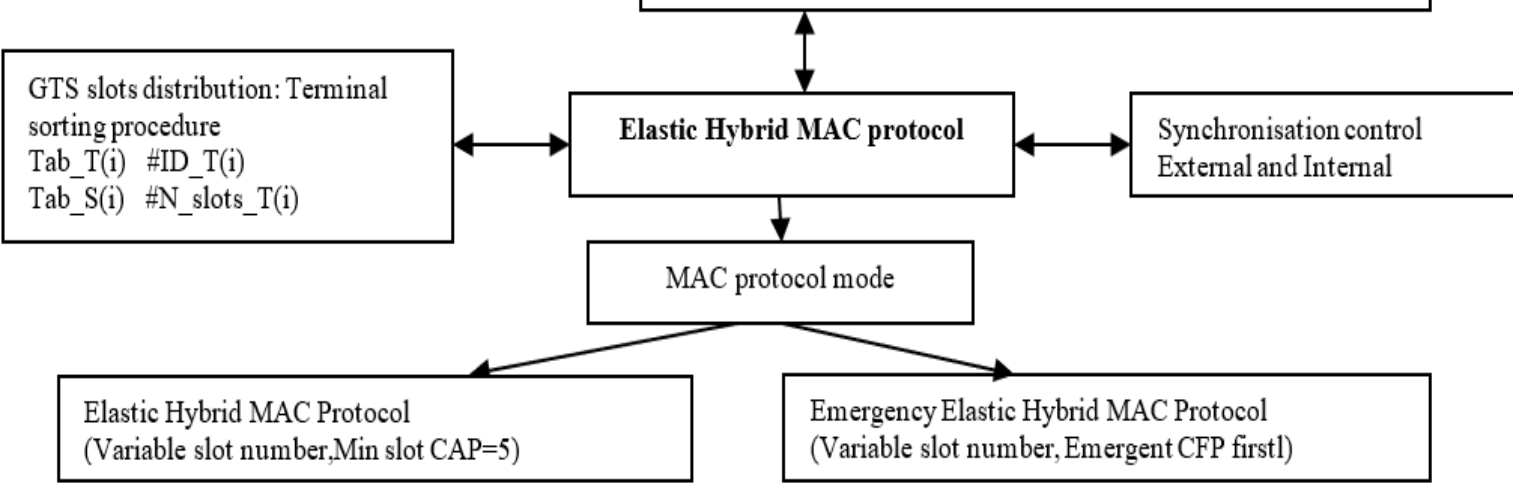

Figure 1. Building blocks for elastic hybrid MAC protocol

As explained above, the proposition uses the two main approaches: CSMA/CA-based and TDMAbased. A CSMA/CA-based approach allows nodes to transmit at according to backoff. If collision is detected, it makes transmitting nodes retry after some delays. A TDMA-based approach is to transmit data by assigning different time slots or frequencies to different nodes whose transmissions are urgent. In normal condition nodes may communicate with each other using a CSMA/CA macintosh operating system (MAC) protocol. In case of information priorities related to traffic rate, FIFO state or persistent collision, concerned nodes will transmit their data using reserved slot based on TDMA protocol. The proposed protocol tolerates emergent CFP slot reservation for marked urgent data to transmit directly after the beacon frame.

The work presented herein is limited to the MAC layer. It focuses on a star topology which can be used as a basic building block of a WSN and it can be easily exploited in clustering architecture. A star network topology considered uses $\mathrm{N}$ nodes varying from 5 to $\mathrm{N}=25$ and a single network coordinator. Each node transmits frames to the coordinator respecting application constraints. We assume that the hybrid MAC protocol operates with a physical data rate of $250 \mathrm{Kbps}$. Time is divided into superframes. The superframe structure is similar to the standard IEEE 802.15.4 superframe structure. The active part of the superframe is divided initially into timeslots allocating 10 slots for CAP and 5 for CFP. Frames are repetitive and are often begun with a broadcast beacon to denote the start of a frame and provide nodes necessary information about slots distribution and synchronization. Each superframe has a contention period graduated on sub-slot backoff equal to $320 \mathrm{~ns}$ and represents the smallest unit of time. We consider two channels for control and data separately to permit a coordinator sending superframe structure information as soon as possible in the next beacon and response quickly to network traffic changes. We suppose that every node can transmit one data frame per slot. If there is $n(n \in[0 . . N])$ active nodes, the total number of requested slot is equal to $n$. 
Since the CAP period represent low priorities nodes, Slots allocated to CAP period are calculated as a subtraction result of active slots and CFP needs. So, the hybrid protocol control continuously the data transfer requirements and then decide to vary schedule and contention slots number according to the necessity designed by traffic conditions. Generally, GTS slots are assigned according to the amount of traffic as considered for a hybrid protocol based in PSM mode and Slotted aloha proposed in [32]. We consider in this work that GTS slots of node $n_{i}$ are allocated based on four factors: traffic rate, first in first out (FIFO) state, persistent collision and emergency case. Figure 2 presents the GTS slots reservation strategy. Synchronization purpose is performed at the beacon slot to maintain a common notion of time across the network. Synchronization is very important when shifting the mode from CSMA to TDMA and vice versa. Then, additional information (position to begin sending allocated slot of a node) is placed on beacon frame to avoid synchronization errors. Therefore, slots dynamic distribution becomes more effective to deal with prioritization in a fluctuate traffic. The elasticity of CAP and CFP periods meets the challenges of efficiently sacrificing the bandwidth without affecting latency constraint and memory state of low priority data nodes.

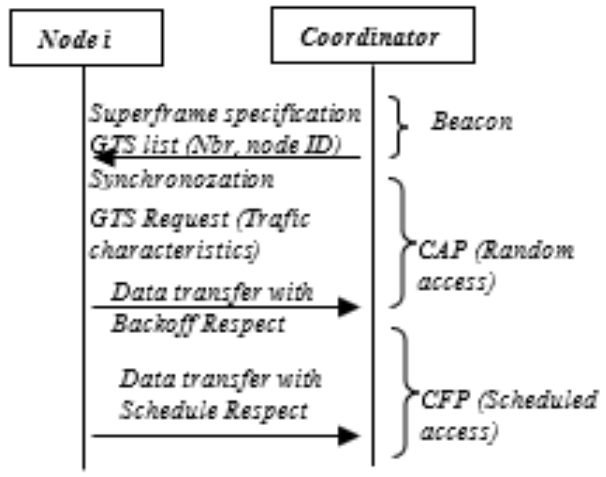

At each Beacon interval:
For each nodecalculate
GTS slots needed fct(rate, emergency,..)
\[ =3,2 \text { or } 1 \text { or (wait for } \]
next interval)
If calculated GTS slots > GTS s lots then
CFP++
Respect CAPmin length
Get GTS slot, @ node, start position
Broadcast beacon frame

Figure 2. GTS reservation strategy and dynamic CAP period adjustment

\section{SIMULATION RESULTS}

Simulation parameters considered in the present work for one hop distance includes multiple sources nodes attached to coordinator node by star topology. Sensors nodes are defined with diverse traffic condition (such as periodic, burst, random, low, and dense traffics) and randomly distributed over time in order to test the reliability and the realistic behaviour of the proposed model. Worst cases are, particularly, established with increasing traffic and increasing collisions to test the quality of system reactions. Proposed protocol makes reasonable priorities for heterogeneous traffic nodes to get access to the superframe. The proposal advantage compared to solutions discussed in this paper is that it takes on account several constraints that may influence the precise decision about slot reservation strategy. It is also remarkable that most ideas are implemented by NS simulator (NS) or other similar environments. In this proposal, we suggest modulating access algorithm using very-high hardware description language. The VHDL language allows parallel processes and time sensitive instructions which permit to define specific variables, various processing functions describing particular event and to emulate real scenarios. Then, during scenarios execution parameters progress is observed using trace files. The simulation phase helps to reason about the efficiency of the system's behaviour. To prove the efficiency of the proposed protocol, comparison and evaluation are discussed with the standard protocol for WSN. Figure 3 depicts a respect of data transfer procedure by concerned nodes (node 21 in this example). It also demonstrates synchronization and dynamic distribution of GTS reservation in respect of priorities considered by decisive mechanism.

Packet delivery ratio (PDR) is usually considered for performance evaluation of MAC protocol for WSNs. It is proportional to throughput as it specifies the frame retransmission rate due to a packet drop or collision which affects the maximum throughput of the network. Figure 4(a) demonstrates that the PDR evolution of enhanced hybrid protocol is always higher than the classical protocol (CSMA based protocol). This result shows the proposed protocol better exploits network resources. Figure 4(b) confirms also the network capacity improvement. The proposed hybrid protocol provides better and consistent results than classic protocol. The Figure illustrates that the PDR still higher when increasing a number of nodes. This is explained by the good reaction of the proposed strategy to distribute sufficiently superframe between contention access phase (CAP) and CFP period and a distribution of slots in each interval. MAC protocol in WSNs must be designed to be able to work with a large number of nodes and minimize packet duration by 
avoiding extended backoff time and unused slot. Therefore, the network system must be efficient even if the number of nodes increased.

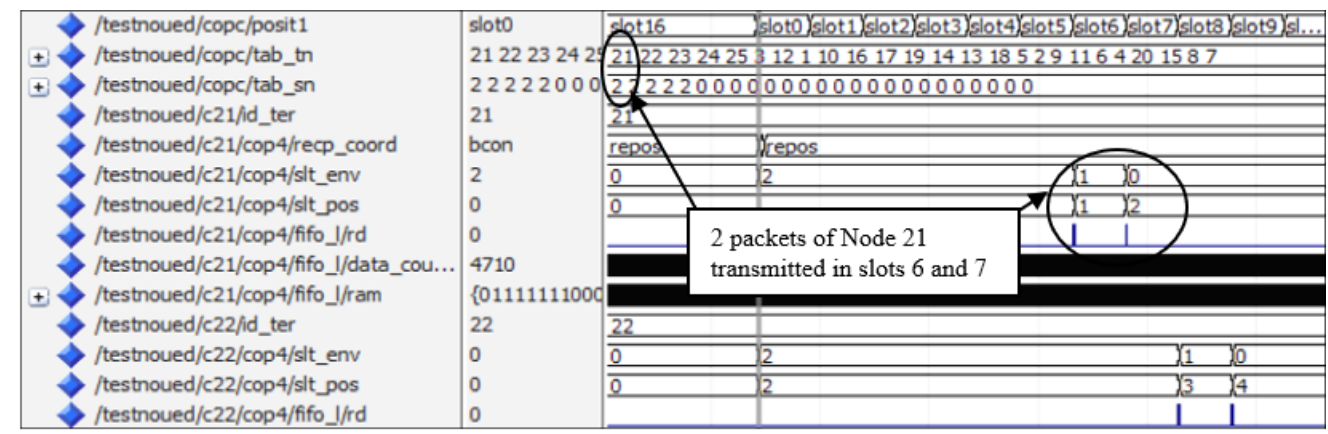

Figure 3. Simulation data transfer in respect of GTS reservation

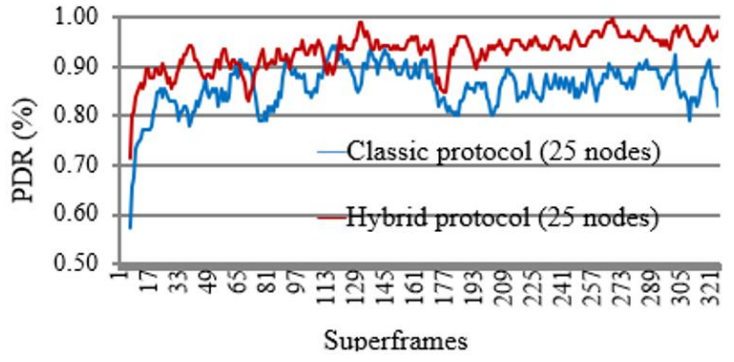

(a)

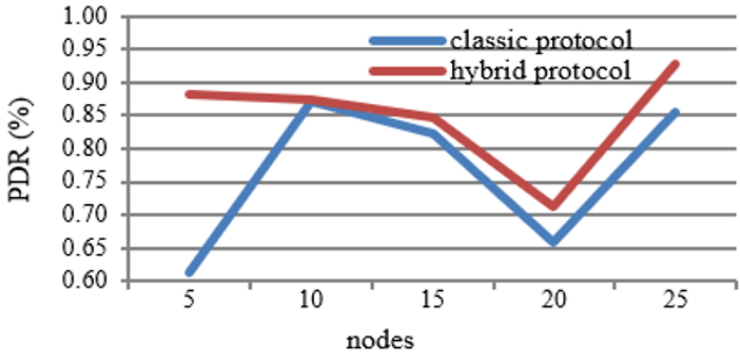

(b)

Figure 4. PDR state comparison, (a) over simulation time, (b) function of number of nodes

Unused slots during the active period of the superframe present a waste of bandwidth. Simulation results show that the elastic hybrid MAC protocol strategy exploits efficiently the active period. It offers 33\% more slot occupation than the standard protocol of IEEE802.15.4 as explained in Figure 5. The consecutive packet losses may cause unreliability of the communication system. Therefore, the packet loss probability is used as another metric to monitor simulation results efficiency. Packet loss reflects a failure of data frame to reach their destination. Packet drop can be caused by queue saturation or discard result of reaching the maximum allowed value of either carrier sensing attempts (CCA) or retransmission attempts. Figure 6 depicts a percentage of packet drop incurred by buffer saturation. The result shows that the E-hybrid protocol decreases a drop probability.

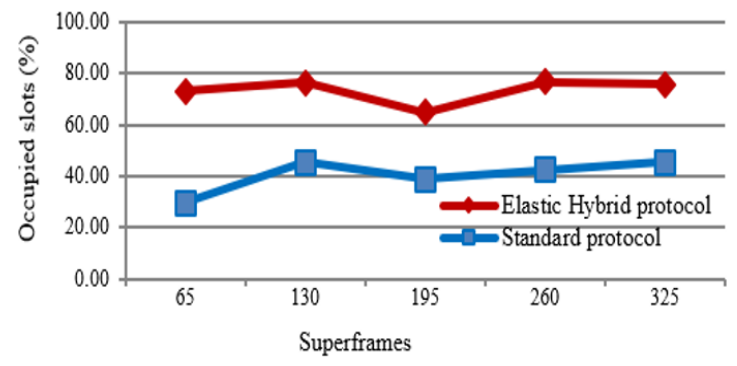

Figure 5. Comparison of occupied slots

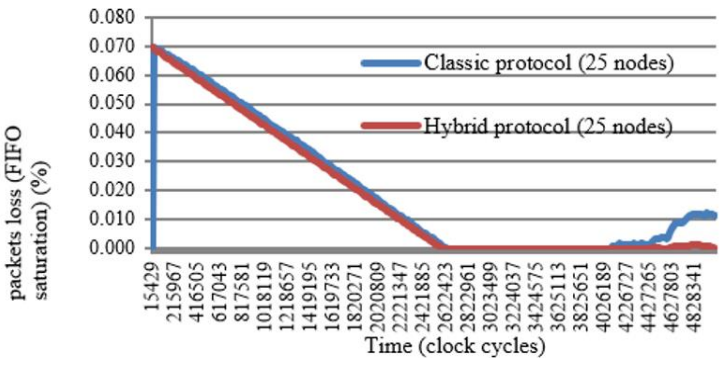

Figure 6. Packet drop function of time simulation

If a node defers the transmission, it saves energy but its buffer level may remain the same or increase. When the node transmits during both CAP and CFP, its throughput increases but it consumes a significant amount of energy. The proposed algorithm tries balancing this compromise based on the 
assumption that nodes with higher buffer occupancy level are unexpected to delay their transmissions and are highly considered to use a TDMA slot during CFP. In consequence, congestion is considerably reduced and FIFO occupancy is remarkably better Figure 7. The elasticity behaviour of the proposed hybrid MAC protocol has a great effect on minimizing congestion rate. Figure 8 shows a decrease of $7 \%$ between classic and proposed protocol.

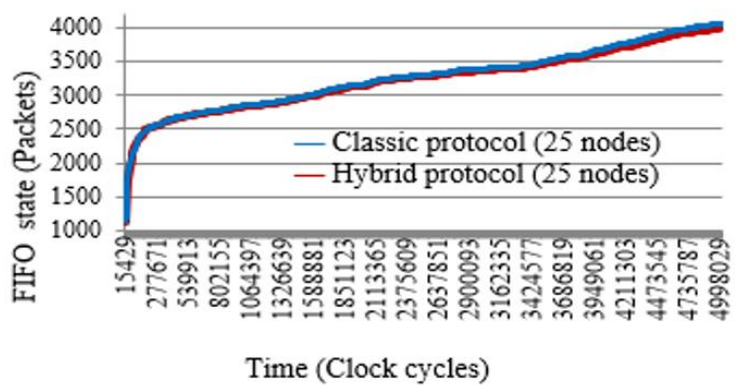

Figure 7. FIFO packets occupancy

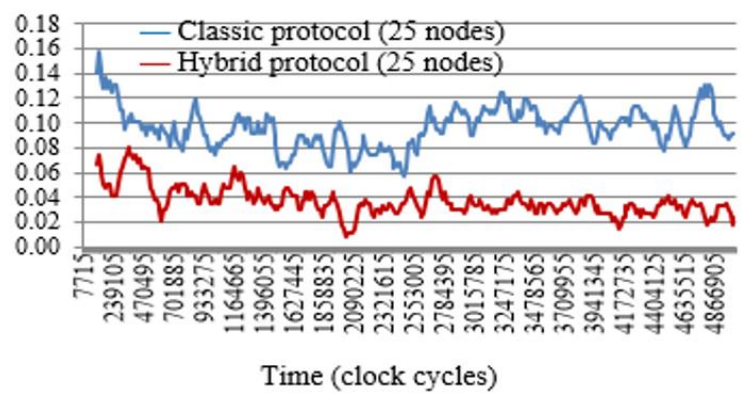

Figure 8. Congestion comparison

\section{CONCLUSION}

The function of WSN is not only monitoring. WSN is usually responsible for more complex operations that need efficient transfer of a large amount of information on time and considering QoS requirements. Combining schedule and contention is advantageous idea. Improvements can be remarkable in gaining energy by decreasing overhead and listening, surmounting problems earned by dense traffic especially the probability of collision, adapted to various traffic conditions and satisfying both scalability and reliability. Allocating TDMA slots dynamically permit attaining full use of the communication bandwidth, establishing balanced bandwidth use, decreasing the transmission delay and keeping transmission synchronization. The elasticity of the hybrid algorithm provides considerable guaranteed transmission, performs network communications, makes timely transfer of emergent data and optimizes QoS. To contribute to further research effort in enhancing MAC protocol of WSNs, open research issues can be investigated to test the presented approach for a larger network based on cluster topology. Then, new challenges not discussed in this paper will be important to be considered in account. These additional traffic control strategies will concern cluster synchronization, routing, mobility and QoS investigation.

\section{REFERENCES}

[1] C. Nicolas, A. M. Ahmad, J. R. Hernan, and G. Habib, "Hybrid Duty Cycle Algorithm for industrial WSNs Using Machine Learning," In book: Machine Learning and Metaheuristics Algorithms, and Applications, Springer 2019, pp. 1-15, doi: 10.1007/978-981-15-4301-2_1.

[2] I. Bouazzi, J. Bhar, and M. Atri, "Priority-based queuing and transmission rate management using a fuzzy logic controller in WSNs," ICT Express Journal 2017/6/1, vol. 3, no. 2, pp. 101-105, doi: 10.1016/j.icte.2017.02.00.

[3] T. Hsieh, K. Lin, and P. Wang, "A hybrid MAC protocol for wireless sensor networks," 2015 IEEE 12th International Conference on Networking, Sensing and Control, Taipei, Taiwan, 2015, pp. 93-98, doi: 10.1109/ICNSC.2015.7116016.

[4] T. Kaur and D. Kumar, "QoS mechanisms for MAC protocols in wireless sensor networks: a survey," in IET Communications, vol. 13, no. 14, pp. 2045-2062, 278 2019, doi: 10.1049/iet-com.2018.5110.

[5] J. Nieminen and R. Jäntti, "Delay-throughput analysis of multi-channel MAC protocols in ad hoc networks," EURASIP Journal on Wireless Communications and Networking 2011, vol. 1, pp. 1-15, 2011, doi: 10.1186/1687-1499-2011-108.

[6] B. Mawlawi, J. Doré, N. Lebedev and J. Gorce, "Multiband CSMA/CA with RTS-CTS strategy," 2014 IEEE 10th International Conference on Wireless and Mobile Computing, Networking and Communications (WiMob), Larnaca, Cyprus, 2014, pp. 628-633, doi: 10.1109/WiMOB.2014.6962236.

[7] B. A Muzakkari, M. A. Mohamed, M. F. A. Kadir, Z. Mohamad, and N. Jamil, "Recent advances in energy efficient-QoS aware MAC protocols for wireless sensor networks," International Journal of Advanced Computer Research, vol. 8, no. 38, pp. 212-228, 2018, doi: doi.org/10.19101/IJACR.2018.837016.

[8] S. Arshad, A. Al-Sadi, and A. Barnawi, "Z-MAC: Performance Evaluation and Enhancements," Procedia Computer Science 21, the fifth International Symposium on Applications of Ad hoc and Sensor Networks, vol. 21, pp. 485-490, 2013, doi: 10.1016/j.procs.2013.09.066. 
[9] T. Zheng, S. Radhakrishnan, and V. Sarangan, "A routing layer sleep scheme for data gathering in wireless sensor networks," 2012 IEEE International Conference on Communications (ICC), Ottawa, ON, Canada, 2012, pp. 735739, doi: 10.1109/ICC.2012.6364364.

[10] T. Zheng, S. Radhakrishnan, and V. Sarangan, "Modeling and performance analysis of DMAC for wireless sensor networks," Proceedings of the 14th International Symposium on Modeling Analysis and Simulation of Wireless and Mobile Systems, MSWiM 2011, Miami, Florida, USA, pp. 119-128, October 31-November 4, 2011, doi: 10.1145/2068897.2068920.

[11] I. Slama, B. Jouaber, and D. Zeghlache, "Priority-Based Hybrid MAC for Energy Efficiency in Wireless Sensor Networks," Wireless Sensor Network, vol. 2, pp. 755-767, 2010, doi: 10.4236/wsn.2010.210091.

[12] C. Venkataramanan and S. M. Girirajkumar, "Hierarchical MAC protocol with adaptive duty-cycle adjustment algorithm for wireless sensor network," International Journal of Operational Research, vol. 37, no. 4, pp. 579-599, 2020, doi: 10.1504/IJOR.2020.105769.

[13] H. Liang, S. Yang, L. Li, and J. Gao, "Research on routing optimization of WSNs based on improved LEACH protocol," EURASIP Journal on Wireless Communications and Networking (2019), p. 194, 2019, doi: 10.1186/s13638-019-1509-y.

[14] J. Lee and T. Kao, "An Improved Three-Layer Low-Energy Adaptive Clustering Hierarchy for Wireless Sensor Networks," in IEEE Internet of Things Journal, vol. 3, no. 6, pp. 951-958, Dec. 2016, doi: 10.1109/JIOT.2016.2530682.

[15] S. Zhuo, Z. Wang, Y. Song, Z. Wang, and L. Almeida, "A Traffic Adaptive Multi-Channel MAC Protocol with Dynamic Slot Allocation for WSNs," in IEEE Transactions on Mobile Computing, vol. 15, no. 7, pp. 1600-1613, July 2016, doi: 10.1109/TMC.2015.2473852.

[16] J. Bhar, "A Mac protocol implementation for wireless sensor network," Journal of computer networks and communications, vol. 2015, no. 697153, p. 12, doi: doi.org/10.1155/2015/697153.

[17] K. Pattamasiriwat and C. Jaikaeo, "Evaluation of low power listening MAC protocol on network monitoring in wireless sensor networks," 2017 9th International Conference on Knowledge and Smart Technology (KST), Chonburi, 2017, pp. 260-265, doi: 10.1109/KST.2017.7886117.

[18] B. Shrestha, E. Hossain, and K. W. Choi, "Distributed and Centralized Hybrid CSMA/CA-TDMA Schemes for Single-Hop Wireless Networks," in IEEE Transactions on Wireless Communications, vol. 13, no. 7, pp. 4050-4065, July 2014, doi: 10.1109/TWC.2014.2327102.

[19] P. Raja and P. Dananjayan, "A Game Theory-Based Hybrid Medium Access Control Protocol for Congestion Control in Wireless Sensor Networks," Game Theoretic Analysis of Congestion, Safety and Security, Springer, Cham, 2015, pp. 1-25, doi: 10.1007/978-3-319-13009-5_1.

[20] L. Wu, S. Zhuo, Z. Wang, and Z. Wang, "pQueue- MAC: An Energy Efficient Hybrid MAC Protocol for EventDriven Sensor Networks," International Journal of Distributed Sensor Networks, vol. 11, vol. 2, 2015, doi: 10.1155/2015/160167.

[21] X. Yang, L. Wang, J. Xie, and Z, Zhang, "Energy Efficiency TDMA/CSMA Hybrid Protocol with Power Control for WSN," Wireless Communications and Mobile Computing, vol. 2018, 2018, doi: doi.org/10.1155/2018/4168354.

[22] E. E. Petrosky, A. J. Michaels, and J. M. Ernst, "A Low Power IoT Medium Access Control for Receiver-Assigned CDMA," presented at the World Telecommunications Symposium (WTS) 2018, Phoenix, Arizona, USA, 2018, doi: 10.4018/IJITN.2019040103.

[23] Y. Z. Zhao, C. Miao, and M. Ma, "A Survey and Projection on Medium Access Control Protocols for Wireless Sensor Networks, "ACM Computing Surveys, vol. 45, no. 1, November 2012, doi: $10.1145 / 2379776.2379783$.

[24] A. Djimli, S. Merniz, and S. Harous, "Energy-efficient MAC protocols for wireless sensor networks: a survey," TELKOMNIKA Telecommunication, Computing, Electronics and Control, vol. 17, no. 5, pp. 2301-2312, October 2019, doi: 10.12928/TELKOMNIKA.v17i5.12163.

[25] I. Iala, I. Dbibih, O. Zytoune, M. Rziza, and D. Aboutajdine, "A Kalman Filter Process for Energy Optimization in WSNs," Journal of Communications Software And Systems, vol. 15, no. 1, March 2019, doi: 10.24138/jcomss.v15i1.561.

[26] M. Ezhilarasi and V. Krishnaveni, "A Survey on Wireless Sensor Network: Energy and Lifetime Perspective," 2018 Swansea Printing Technology Ltd Taga Journal, vol. 14, pp. 3099-3113, 2018.

[27] M. Ghribi and A. Meddeb, "Survey and taxonomy of MAC, routing and cross layer protocols using wake-up radio," Journal of Network and Computer Applications, vol. 149, 2020, Art. No. 102465, doi: 10.1016/j.jnca.2019.102465.

[28] M. Gamal, N. Sadek, M. R. M. Rizk, M. Abd ElAzim, "Optimization and modeling of modified unslotted CSMA/CA for wireless sensor networks," Alexandria Engineering Journal, vol. 59, pp. 681-691, 2020, doi: 10.1016/j.aej.2020.01.035.

[29] A. S. Althobaiti1 and M. Abdullah, "Medium Access Control Protocols for Wireless Sensor Networks Classifications and Cross-Layering," Procedia Computer Science 65 (2015) 4 - 16, International Conference on Communication, Management and Information Technology (ICCMIT 2015), doi: 10.1016/j.procs.2015.09.070.

[30] A. Kumar, M. Zhao, K. Wong, Y. L. Guan and P. H. J. Chong, "A Comprehensive Study of IoT and WSN MAC Protocols: Research Issues, Challenges and Opportunities," in IEEE Access, vol. 6, pp. 76228-76262, 2018, doi: 10.1109/ACCESS.2018.2883391.

[31] A.A. Khan, S. Ghani, and S. Siddiqui, "A Taxonomy for MAC Protocols in Wireless Sensor Networks Based on Traffic Prioritization," Wireless Pers Commun, vol. 104, pp. 1493-1522, 2019, doi: 10.1007/s11277-018-6095-5. 
[32] S. Mehta and K. S. Kwak, "H-MAC: A hybrid MAC protocol for wireless sensor networks," International journal of Computer Networks and Communications vol. 2, no. 2, March 2010, doi: 10.5121/ijenc.2010.2208.

\section{BIOGRAPHIES OF AUTHORS}

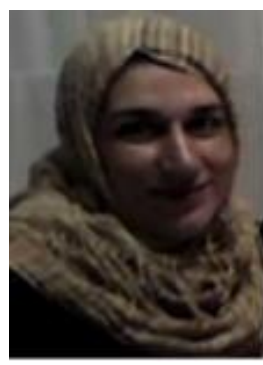

Jamila Bhar received her doctoral degree in Electric and Communication system from the National School of Engineering of Sfax (ENIS), Tunisia in 2010. Currently she is a member of the NOCCS Laboratory in ENISo, Sousse - Tunisia. Her research interests include Hardware/software protocol design and verification, Traffic management, Handover protocol and Quality of Service for wireless Sensor Networks and Fuzzy Logic.

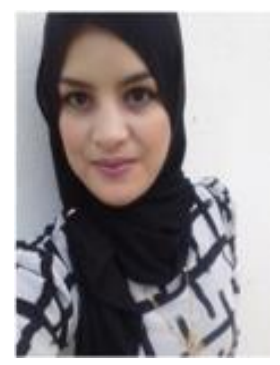

Imen Bouazzi obtained her $\mathrm{PhD}$ degree in Science and technology from the Faculty of Science in Monastir, Tunisia in 2018; she obtained her engineering degree in Applied Science in Technology (speciality - Electronic Microelectronics, July 2013) from the higher institute of Computer and Mathematical of Monastir. Her interest's research is concentrated on computer science, network communication and electronic wireless devices. 